\title{
Regulation of VEGF-A Expression in Endothelial Cells by Transcriptional Gene Activation or Transcriptional Gene Silencing: Analysis of Genome Wide Transcriptional Response
}

\section{Tiia Husso ${ }^{1 \#}$, Mikko P. Turunen ${ }^{1 \#}$ and Seppo Yla-Herttuala ${ }^{1,2 *}$}

${ }^{1}$ Department of Biotechnology and Molecular Medicine, A.I.Virtanen Institute, University of Eastern Finland, Finland

${ }^{2}$ Science Service Center and Gene Therapy Unit, Kuopio University Hospital, Kuopio, Finland

\# Equal Contribution

\begin{abstract}
Vascular endothelial growth factor (VEGF-A) is an important gene in many diseases, such as cancer and cardiovascular diseases. We investigated changes in genome-wide gene expression patterns in murine endothelial cells when the expression of VEGF-A was epigenetically modulated using promoter targeted small hairpin RNAs (shRNAs). Murine endothelial cells were transduced with lentiviral vectors expressing shRNAs that are complementary to the gene promoter. Gene expression array experiments were conducted to investigate genome-wide mRNA expression changes caused by up- and downregulating VEGF-A. There were several hundreds of differentially expressed genes according to the applied statistical criteria. We noticed that the effects of downregulating VEGF-A were much more wide-spread than the effects of VEGF-A upregulation. Our in silico analysis revealed that a number of different biological processes are altered due to epigenetic effects on VEGF-A expression. One of the main regulators of VEGF-A mediated transcriptional response was found to be transcription factor ATF-4. This is the first study showing the transcriptional response to epigenetic modification of VEGF-A expression in endothelial cells. Epigenetic gene regulation represents a natural gene regulatory mechanism and these results reveal previously unknown implications of VEGF-A regulation in endothelial cells.
\end{abstract}

Keywords: Transcriptional gene silencing; Transcriptional gene activation; Endothelial cell; Microarray

\section{Introduction}

Vascular endothelial growth factor (VEGF-A) is a key regulator of angiogenesis in many diseases, such as cancer and cardiovascular diseases [1]. For that reason it is not surprising that its effects on endothelial cells have been studied by several approaches, such as microarray analysis [2] and next generation sequencing [3]. For example, Shin et al. used microarray analysis to study transcriptional response to human dermal lymphatic endothelial cells treated with recombinant human VEGF-A165 [4]. For the inhibition of VEGF/ VEGFR signaling, Bevacizumab has been used in a mouse xenograft model of endometrial cancer [5]. However, manipulation of VEGF-A expression, either upregulating the expression by delivering VEGF-A expression cassettes and recombinant proteins or inhibiting it by using RNAi or antibodies, is somewhat artificial in terms of endothelial biology. Recently it has become evident that small RNAs, such as miRNA, siRNA or shRNA, can regulate target gene expression via promoter targeted epigenetic modifications [6-8]. These processes have been studied in plants for a long time, but when Morris et al. [6] found that small RNAs directed to the gene promoters induce transcriptional gene silencing (TGS) and a little later Li et al. [7] showed that they can also induce transcriptional gene activation (TGA), their potential as therapeutics was quickly realized. However, the specificity and safety of these promoter targeted small RNAs must be evaluated before clinical trials.

Modulating the expression levels of mouse VEGF-A was achieved by transducing mouse endothelial cells with lentiviral vectors that express different shRNAs. These molecules are complementary to the promoter of mouse VEGF-A gene, and they are known to either increase or decrease VEGF-A expression by causing epigenetic changes in the promoter region as described previously $[8,9]$. Epigenetic gene regulation is a natural mechanism for altering gene expression during development and adaptation to new situations. In this study, we analyzed gene expression responses in endothelial cells after epigenetic manipulation of VEGF-A and bioinformatically predicted possible offtarget effects of these shRNAs. This data is important for the future development of this technique for clinical gene therapy.

\section{Methods}

An expanded Materials and Methods section is available in the Data Supplement.

\section{Vector constructs}

The construction of lentiviral (LV) shRNA expression vectors has been described [8]. The used target sequences were: CGTTCTCAGTGCCACAAAT (-856) and GACGCGTGTTTCAATGTGA $(-451)$. The number in parenthesis refers to the first nucleotide in the sequence of the shRNA relative to the TSS of mouse VEGF-A promoter (U41383). As a control we used LV encoding only green fluorescent protein (GFP) without a shRNA cassette. The third generation HIV-1 based LV-PGK-GFP-U6shRNA vectors were prepared by standard calcium phosphate transfection method in 293T cells as described [10].

\section{Transductions, sample preparation and Affymetrix analysis}

C166 cells were transduced with lentiviral vector expressing either

*Corresponding author: Seppo Yla-Herttuala, A.I.Virtanen Institute for Molecular Sciences, University of Eastern Finland, P.O. Box 1627, FIN-70211 Kuopio, Finland, Tel: 00202 2683147; E-mail: seppo.ylaherttuala@uef.fi

Received May 13, 2015; Accepted June 01, 2015; Published June 03, 2015

Citation: Husso T, Turunen MP, Yla-Herttuala S (2015) Regulation of VEGF-A Expression in Endothelial Cells by Transcriptional Gene Activation or Transcriptional Gene Silencing: Analysis of Genome Wide Transcriptional Response. Gene Technology 4: 122. doi: 10.4172/2329-6682.1000122

Copyright: $(2015$ Husso T, et al. This is an open-access article distributed under the terms of the Creative Commons Attribution License, which permits unrestricted use, distribution, and reproduction in any medium, provided the original author and source are credited. 
Citation: Husso T, Turunen MP, Yla-Herttuala S (2015) Regulation of VEGF-A Expression in Endothelial Cells by Transcriptional Gene Activation or Transcriptional Gene Silencing: Analysis of Genome Wide Transcriptional Response. Gene Technology 4: 122. doi: 10.4172/23296682.1000122

upregulating shRNA, downregulating shRNA, or LV control (referred in this report as "VEGF-A UP", "VEGF-A DOWN" and control, respectively). Cells were transduced at $20 \%$ confluency using MOI 10 and samples were collected 7 days after the transduction. Sample preparation, hybridization and scanning were all done using standard Affymetrix protocols. The gene expression patterns of each group of cells were measured with 4 expression arrays (Affymetrix GeneChip Mouse Genome 4302.0 Array), totaling 12 arrays.

\section{Statistical Analysis}

After preprocessing there were expression data for 17370 genes. From this set, we identified and filtered out those expression profiles where all absolute expression levels were in the lowest 10 percent of the data set. Statistical scores for these genes would be too inaccurate. 1518 genes were excluded based on this criterion. The data were then divided into three sets of four arrays each: downregulation, upregulation and control experiments. For each gene, we computed two fold changes and two differential expression p-values corresponding to "VEGF-A DOWN" experiment vs. control and "VEGF-A UP" experiment vs. control. P-values were computed with two-tailed t-test with permutation testing (10,000 permutations). To correct for multiple hypothesis testing related errors, we computed false discovery rates (FDR) using the Storey-Tibshirani procedure [11]. False discovery rate is defined as the expected ratio of the number of false positives to the total number of positive calls in a differential expression analysis between two groups of samples. The q-values, which measure the minimum FDR that occurs when calling the test significant, were also computed.

A gene is here considered to be differentially expressed if it shows both statistical and biological significance. Using $\mathrm{p}$-values as a measure of statistical significance $(\mathrm{p}<0.01)$ and fold change $(\mathrm{FC})(\log 2(\mathrm{FC})>0.5$ for the "VEGF-A DOWN" experiment, and $\log 2(\mathrm{FC})>0.25$ for the "VEGF-A UP" experiment) as a measure of biological significance, the differentially expressed genes were computed for both experiments. A more stringent fold change criterion was applied in the "VEGF-A DOWN" experiment because these expression profiles deviated considerably more from the control profiles.

\section{Gene ontology and pathway analysis}

Gene ontologies, pathways affected and regulatory transcription factors were analyzed using Ingenuity Pathway Analysis tool, (IPA, www.ingenuity.com) by analyzing only the datasets of genes that we found significantly regulated by our own analysis.

\section{qRT-PCR}

cDNA synthesiswas performed using Transcriptor FirstStrandcDNA Synthesis Kit (Roche) according to the manufacturer's instructions. Real-time quantitative PCR was performed with a LightCycler 480 apparatus (Roche) using TaqMan Gene Expression Assays (Applied Biosystems) for ACTB (4352663), Tnnt2 (Mm01290256_m1), Kdr (Mm004401111_m1), Vegfa (Mm00437306_m1), Ccl2 (Mm00441242_ m1), Selp (Mm00441295_m1), Mmp13 (Mm00439491_m1), Angptl4 (Mm00480431_m1) and LightCycler 480 Probes Master (Roche). PCR cycling conditions were: $10 \mathrm{~min}$ at $95^{\circ} \mathrm{C}$, followed by 50 cycles of $15 \mathrm{~s}$ at $95^{\circ} \mathrm{C}$ and $60 \mathrm{~s}$ at $60^{\circ} \mathrm{C}$. Fold changes were calculated using the formula 2- $(\Delta \Delta \mathrm{Ct})$, where $\Delta \Delta \mathrm{Ct}=\Delta \mathrm{Ct}(\operatorname{shRNA})-\Delta \mathrm{Ct}(\mathrm{GFP})$, and $\Delta \mathrm{Ct}=\mathrm{Ct}$ (Target gene) $-\mathrm{Ct}(\mathrm{ACTB})$. Ct is the cycle at which the threshold line is crossed.

\section{Results}

Based on the selected criteria, there were 326 overexpressed genes and 469 downregulated genes in the "VEGF-A UP" experiment; and
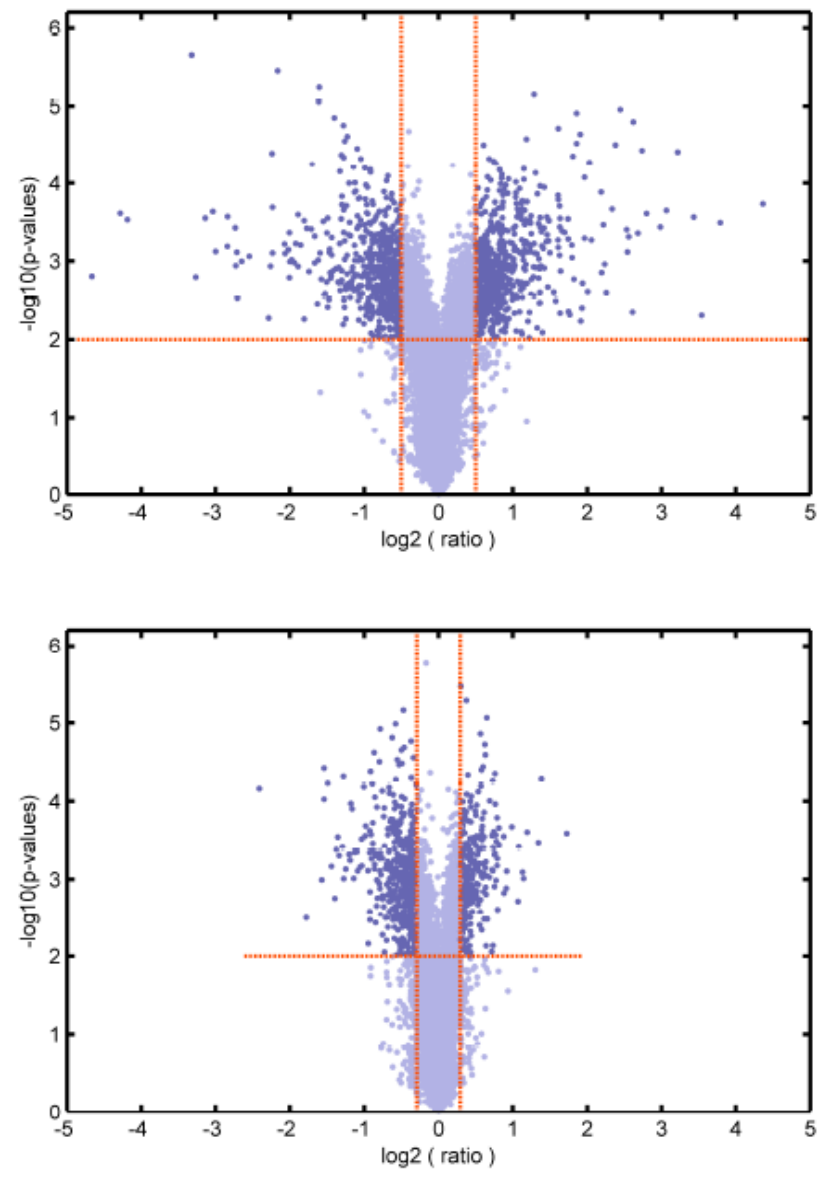

Figure 1: Volcano plots. Upper panel shows a volcano plot for the downregulation experiment and lower panel for the upregulation experiment. The log-ratio of mean gene expression is plotted on the $x$-axis and negative logarithm of $p$-value is plotted on the $y$-axis. Orange dotted lines correspond to the $p$ value threshold $(p<0.01)$ and fold change threshold $(0.5$ for downregulation experiment and 0.25 for upregulation experiment). Darker blue dots, which exceed both thresholds, are significantly differentially expressed genes, and light blue dots that fail to exceed either threshold are not differentially expressed.

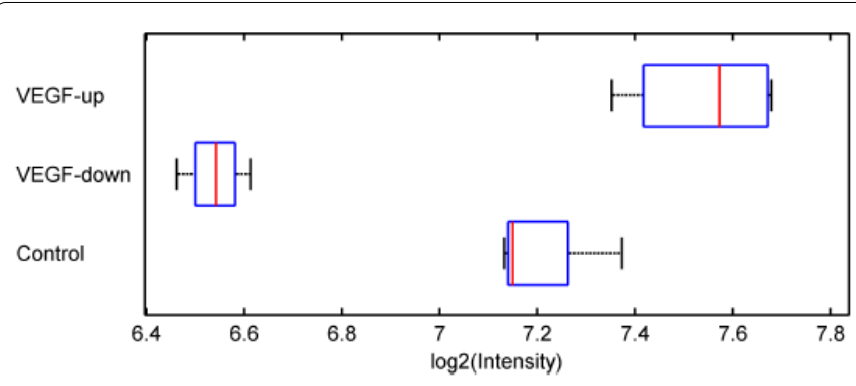

Figure 2: Boxplot of VEGF-A log-intensities. VEGF-A expression is clearly elevated in the upregulation experiment (VEGF-up) compared to the control group (Control). Similarly, the expression of VEGF-A is significantly repressed in the downregulation experiment (VEGF-down). Red lines mark median values, edges of the boxes are the 25th and 75th percentiles, and the whiskers extend to the most extreme data points.

618 overexpressed genes and 587 downregulated genes in the "VEGF-A DOWN" experiment. In Figure 1, volcano plots for both experiments are shown. The spread of data points in the volcano plot is notably wider in the "VEGF-A DOWN" experiment, indicating a more widespread 
Citation: Husso T, Turunen MP, Yla-Herttuala S (2015) Regulation of VEGF-A Expression in Endothelial Cells by Transcriptional Gene Activation or Transcriptional Gene Silencing: Analysis of Genome Wide Transcriptional Response. Gene Technology 4: 122. doi: 10.4172/23296682.1000122

effect of VEGF-A modulation.

In Figure 2, VEGF-A intensities are plotted for each of the three experiments. There is a marked downregulation of VEGF-A in the downregulation experiment and a more modest upregulation of VEGF-A in the upregulation experiment. In Figure 3, we performed hierarchical clustering (with Euclidean distance and Ward's linkage) to show that the arrays cluster correctly together according to the experiments.

It can be noted that arrays from the upregulation experiment cluster more closely with the control experiments, which means that the genome-wide effects of the downregulating shRNA are stronger. The relative expression levels of the differentially expressed genes were hierarchically clustered to investigate the proportions of up- and downregulated genes in the two experiments (Figure 4).

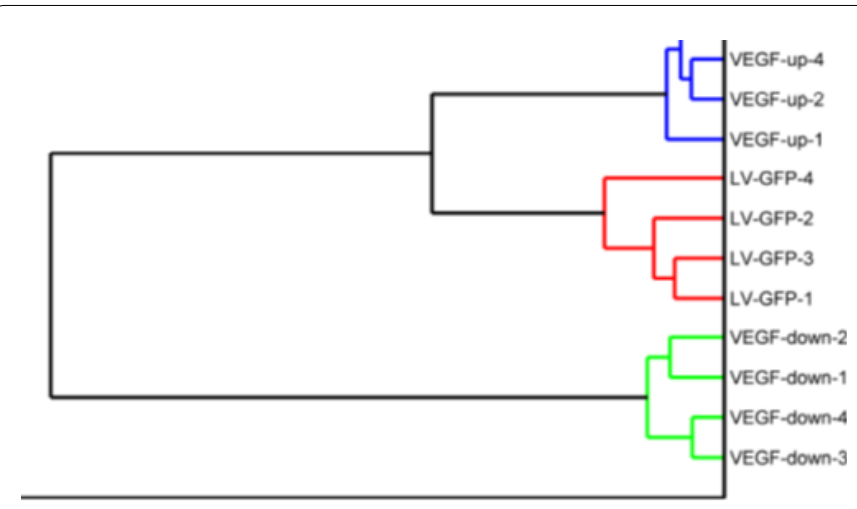

Figure 3: Dendrogram plot of hierarchical cluster tree of arrays. Arrays were hierarchically clustered (with Euclidean distance and Ward's linkage) to examine the similarities between samples. All arrays cluster tightly according to the experiment. Noteworthy, however, is that arrays from the upregulation experiment (blue cluster) seem to be considerably more similar to the controls (red) than arrays from the downregulation experiment (green). This indicates that the effects of VEGF-A downregulation are more widespread than the effects of upregulation.

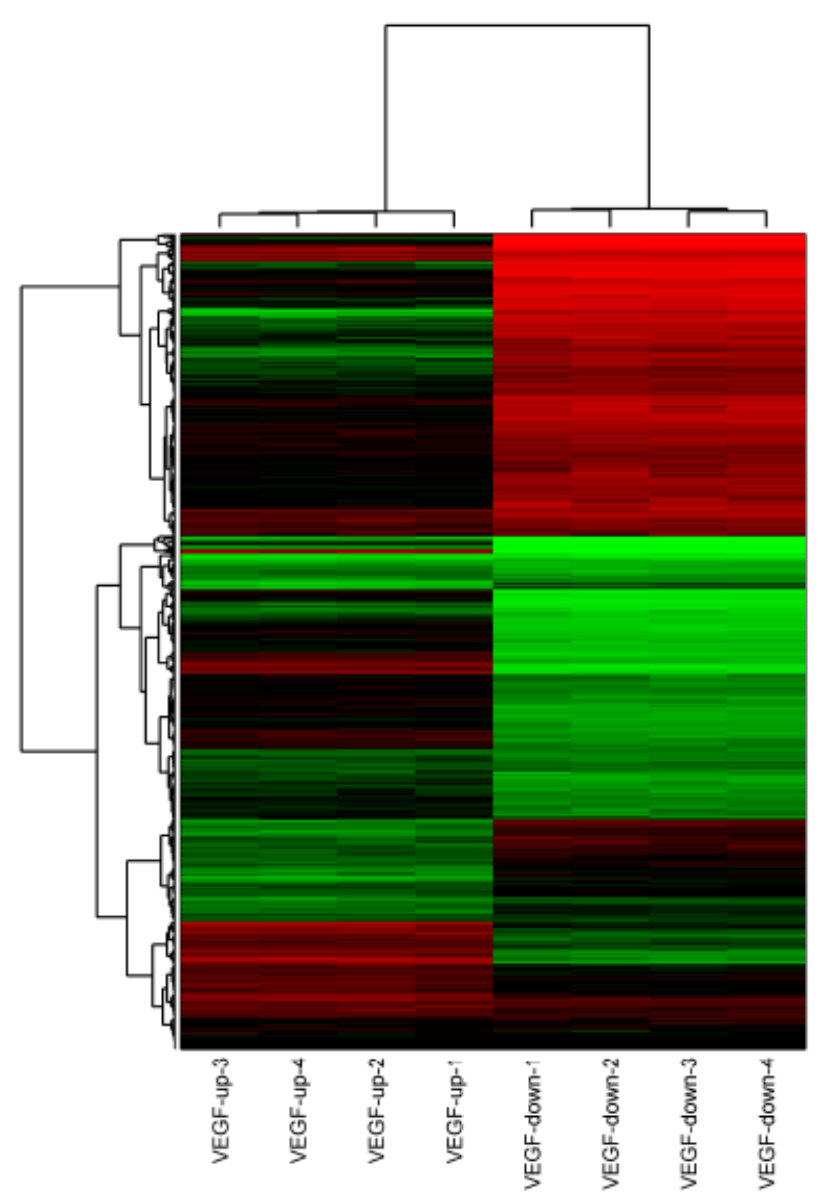

Figure 4: Clustered expression ratios of differentially expressed genes. Expression of each gene was normalized against the mean of control samples and then hierarchically clustered (with Euclidean distance and Ward's linkage) to reveal the patterns of differential expression. There are significantly more over- (red) and underexpressed (green) genes in the downregulation experiment (VEGF-down) compared to the samples from the upregulation experiment (VEGF-up). Interestingly, there are also a few genes that are either repressed or elevated in both of the experiments.

\begin{tabular}{|c|c|c|c|c|c|c|}
\hline Entrez ID & Gene & Fold change & T-statistic & P-value & FDR & Q-value \\
\hline 14955 & $\mathrm{H} 19$ & $-25,50$ & 10,5 & 0,0017 & 0,0040 & 0,0040 \\
\hline 12353 & Car6 & $-18,86$ & 33,8 & 0,0001 & 0,0039 & 0,0038 \\
\hline 21956 & Tnnt2 & $-18,58$ & 27,6 & 0,0002 & 0,0040 & 0,0039 \\
\hline 21952 & Tnni1 & $-10,71$ & 11,1 & 0,0015 & 0,0040 & 0,0040 \\
\hline 216188 & Aldh1/2 & $-10,05$ & 93,6 & 0,0000 & 0,0020 & 0,0020 \\
\hline 50706 & Postn & $-8,83$ & 20,8 & 0,0003 & 0,0040 & 0,0039 \\
\hline 107869 & Cth & $-8,31$ & 27,0 & 0,0002 & 0,0040 & 0,0039 \\
\hline 17022 & Lum & $-8,13$ & 14,6 & 0,0008 & 0,0040 & 0,0040 \\
\hline 20210 & Saa3 & $-7,18$ & 18,4 & 0,0005 & 0,0040 & 0,0040 \\
\hline 108151 & Sema3d & $-7,10$ & 29,2 & 0,0001 & 0,0039 & 0,0039 \\
\hline 11607 & Agtr1a & $-6,77$ & 7,4 & 0,0029 & 0,0042 & 0,0042 \\
\hline 70726 & Angpt|6 & $-6,66$ & 21,3 & 0,0003 & 0,0040 & 0,0039 \\
\hline 66695 & Aspn & $-6,55$ & 15,8 & 0,0007 & 0,0040 & 0,0040 \\
\hline 20319 & Sfrp2 & $-6,40$ & 13,3 & 0,0010 & 0,0040 & 0,0040 \\
\hline 14734 & Gpc3 & $-6,20$ & 13,8 & 0,0009 & 0,0040 & 0,0040 \\
\hline 18787 & Serpine1 & $-6,01$ & 17,2 & 0,0006 & 0,0040 & 0,0040 \\
\hline 66246 & Osgep & $-4,83$ & 13,1 & 0,0010 & 0,0040 & 0,0040 \\
\hline 654812 & Angptl7 & $-4,74$ & 15,7 & 0,0007 & 0,0040 & 0,0040 \\
\hline 70544 & 5730437N04Rik & $-4,74$ & 46,0 & 0,0000 & 0,0037 & 0,0037 \\
\hline 17844 & Mup5 & 4,99 & $-10,7$ & 0,0016 & 0,0040 & 0,0040 \\
\hline
\end{tabular}


Citation: Husso T, Turunen MP, Yla-Herttuala S (2015) Regulation of VEGF-A Expression in Endothelial Cells by Transcriptional Gene Activation or Transcriptional Gene Silencing: Analysis of Genome Wide Transcriptional Response. Gene Technology 4: 122. doi: 10.4172/23296682.1000122

Page 4 of 8

\begin{tabular}{|c|c|c|c|c|c|c|}
\hline 64929 & Scel & 5,04 & $-36,8$ & 0,0001 & 0,0039 & 0,0038 \\
\hline 22403 & Wisp2 & 5,44 & $-55,7$ & 0,0000 & 0,0036 & 0,0036 \\
\hline 27226 & Pla2g7 & 5,62 & $-17,3$ & 0,0005 & 0,0040 & 0,0040 \\
\hline 234564 & Ces1f & 5,72 & $-20,0$ & 0,0004 & 0,0040 & 0,0040 \\
\hline 17178 & Fxyd3 & 6,10 & $-21,8$ & 0,0003 & 0,0040 & 0,0039 \\
\hline 56047 & Msln & 6,16 & $-51,0$ & 0,0000 & 0,0037 & 0,0037 \\
\hline 116872 & Serpinb7 & 6,56 & $-56,7$ & 0,0000 & 0,0036 & 0,0036 \\
\hline 235345 & 4833427G06Rik & 6,65 & $-21,6$ & 0,0003 & 0,0040 & 0,0039 \\
\hline 15245 & Hhip & 6,96 & $-33,2$ & 0,0001 & 0,0039 & 0,0039 \\
\hline 108096 & Slco1a5 & 7,35 & $-4,9$ & 0,0056 & 0,0048 & 0,0048 \\
\hline 20527 & Slc2a3 & 7,38 & $-107,1$ & 0,0000 & 0,0000 & 0,0000 \\
\hline 13107 & Cyp2f2 & 7,69 & $-20,2$ & 0,0004 & 0,0040 & 0,0039 \\
\hline 108078 & Olr1 & 8,37 & $-24,5$ & 0,0002 & 0,0040 & 0,0039 \\
\hline 77055 & Krt76 & 9,13 & $-21,9$ & 0,0003 & 0,0040 & 0,0039 \\
\hline 16691 & Krt8 & 10,20 & $-4,9$ & 0,0056 & 0,0048 & 0,0048 \\
\hline 16542 & Kdr & 10,52 & $-43,5$ & 0,0000 & 0,0038 & 0,0038 \\
\hline 56811 & Dkk2 & 12,95 & $-17,5$ & 0,0005 & 0,0040 & 0,0040 \\
\hline 21950 & Tnfsf9 & 19,76 & $-28,3$ & 0,0001 & 0,0040 & 0,0039 \\
\hline 270097 & Vat1I & 34,74 & $-26,3$ & 0,0002 & 0,0040 & 0,0039 \\
\hline
\end{tabular}

Table 1: Twenty most downregulated and twenty most upregulated genes in response to downregulation of VEGF-A by TGS.

\begin{tabular}{|c|c|c|c|c|c|c|}
\hline Entrez ID & Gene & Fold change & T-statistic & P-value & FDR & Q-value \\
\hline 386463 & Cdsn & 3,26 & $-13,2$ & 0,0003 & 0,0103 & 0,0103 \\
\hline 12353 & Car6 & 2,62 & $-20,4$ & 0,0001 & 0,0100 & 0,0100 \\
\hline 66222 & Serpinb1a & 2,57 & $-12,5$ & 0,0003 & 0,0105 & 0,0104 \\
\hline 110454 & Ly6a & 2,29 & $-13,6$ & 0,0002 & 0,0103 & 0,0103 \\
\hline 17772 & Mtm1 & 2,22 & $-9,2$ & 0,0007 & 0,0109 & 0,0109 \\
\hline 235345 & 4833427G06Rik & 2,13 & $-7,0$ & 0,0013 & 0,0116 & 0,0115 \\
\hline 59083 & Fetub & 2,12 & $-11,1$ & 0,0004 & 0,0106 & 0,0106 \\
\hline 11468 & Actg2 & 2,05 & $-6,7$ & 0,0014 & 0,0117 & 0,0116 \\
\hline 20706 & Serpinb9b & 2,05 & $-53,3$ & 0,0000 & 0,0051 & 0,0051 \\
\hline 13380 & Dkk1 & 2,00 & $-9,3$ & 0,0006 & 0,0109 & 0,0108 \\
\hline 107869 & Cth & 1,92 & $-9,3$ & 0,0006 & 0,0108 & 0,0107 \\
\hline 57875 & Angpt|4 & 1,90 & $-11,3$ & 0,0004 & 0,0107 & 0,0105 \\
\hline 103172 & Chchd10 & 1,88 & $-7,7$ & 0,0010 & 0,0112 & 0,0112 \\
\hline 16669 & Krt19 & 1,82 & $-4,9$ & 0,0030 & 0,0141 & 0,0141 \\
\hline 17386 & Mmp13 & 1,75 & $-5,7$ & 0,0021 & 0,0126 & 0,0126 \\
\hline 228139 & $\mathrm{P} 2 \mathrm{rx} 3$ & 1,75 & $-7,4$ & 0,0011 & 0,0113 & 0,0113 \\
\hline 56772 & Mllt11 & 1,74 & $-8,8$ & 0,0007 & 0,0109 & 0,0109 \\
\hline 69219 & Ddah1 & 1,74 & $-14,7$ & 0,0002 & 0,0102 & 0,0101 \\
\hline 16149 & $\mathrm{Cd} 74$ & 1,74 & $-3,8$ & 0,0068 & 0,0207 & 0,0207 \\
\hline 22041 & Trf & $-2,28$ & 16,6 & 0,0001 & 0,0102 & 0,0100 \\
\hline 15109 & $\mathrm{Hal}$ & $-2,28$ & 10,2 & 0,0005 & 0,0107 & 0,0107 \\
\hline 66695 & Aspn & $-2,29$ & 10,2 & 0,0005 & 0,0107 & 0,0107 \\
\hline 20344 & Selp & $-2,32$ & 23,4 & 0,0000 & 0,0093 & 0,0089 \\
\hline 17392 & Mmp3 & $-2,40$ & 7,7 & 0,0010 & 0,0112 & 0,0112 \\
\hline 11676 & Aldoc & $-2,41$ & 16,4 & 0,0001 & 0,0103 & 0,0100 \\
\hline 20319 & Sfrp2 & $-2,41$ & 9,0 & 0,0007 & 0,0110 & 0,0109 \\
\hline 20309 & Cxcl15 & $-2,41$ & 22,1 & 0,0000 & 0,0098 & 0,0097 \\
\hline 19215 & Ptgds & $-2,50$ & 8,2 & 0,0009 & 0,0111 & 0,0111 \\
\hline 17879 & Myh1 & $-2,55$ & 6,5 & 0,0015 & 0,0117 & 0,0117 \\
\hline 11816 & Apoe & $-2,58$ & 10,3 & 0,0005 & 0,0108 & 0,0107 \\
\hline 11606 & Agt & $-2,59$ & 11,3 & 0,0004 & 0,0106 & 0,0105 \\
\hline 12623 & Ces1g & $-2,60$ & 12,0 & 0,0003 & 0,0106 & 0,0105 \\
\hline 16000 & $\operatorname{lgf} 1$ & $-2,71$ & 8,5 & 0,0008 & 0,0110 & 0,0110 \\
\hline 20296 & $\mathrm{Ccl} 2$ & $-2,89$ & 22,0 & 0,0000 & 0,0099 & 0,0097 \\
\hline 96875 & Prg4 & $-2,90$ & 13,9 & 0,0002 & 0,0103 & 0,0103 \\
\hline 20306 & Ccl7 & $-2,95$ & 18,4 & 0,0001 & 0,0101 & 0,0100 \\
\hline 14955 & $\mathrm{H} 19$ & $-2,99$ & 7,1 & 0,0012 & 0,0115 & 0,0115 \\
\hline 78896 & 1500015010Rik & $-3,20$ & 4,3 & 0,0045 & 0,0164 & 0,0164 \\
\hline 104158 & Ces1d & $-5,37$ & 20,0 & 0,0001 & 0,0100 & 0,0100 \\
\hline
\end{tabular}

Table 2: Twenty most upregulated and twenty most downregulated genes in response to upregulation of VEGF-A by TGA. 
Citation: Husso T, Turunen MP, Yla-Herttuala S (2015) Regulation of VEGF-A Expression in Endothelial Cells by Transcriptional Gene Activation or Transcriptional Gene Silencing: Analysis of Genome Wide Transcriptional Response. Gene Technology 4: 122. doi: 10.4172/23296682.1000122

Page 5 of 8

Clearly, the downregulation experiment yielded in much stronger expression patterns than the overexpression experiment. There are only a few genes that are differentially expressed to the same direction in both experiments. In Tables 1 and 2, the most differentially expressed genes are listed with appropriate statistical scores. Full lists of differentially expressed genes can be found in the supplementary Table 1 and 2.

\section{Down regulation of VEGF-A}

Genes that are linked to cancer (154 genes), cardiovascular disease (83 genes), developmental disorders ( 45 genes), inflammatory response (121 genes) and genetic disorders (67 genes) are significantly regulated in response to the downregulation of VEGF-A in endothelial cells. Top 5 molecular and cellular functions associated are: cell growth and

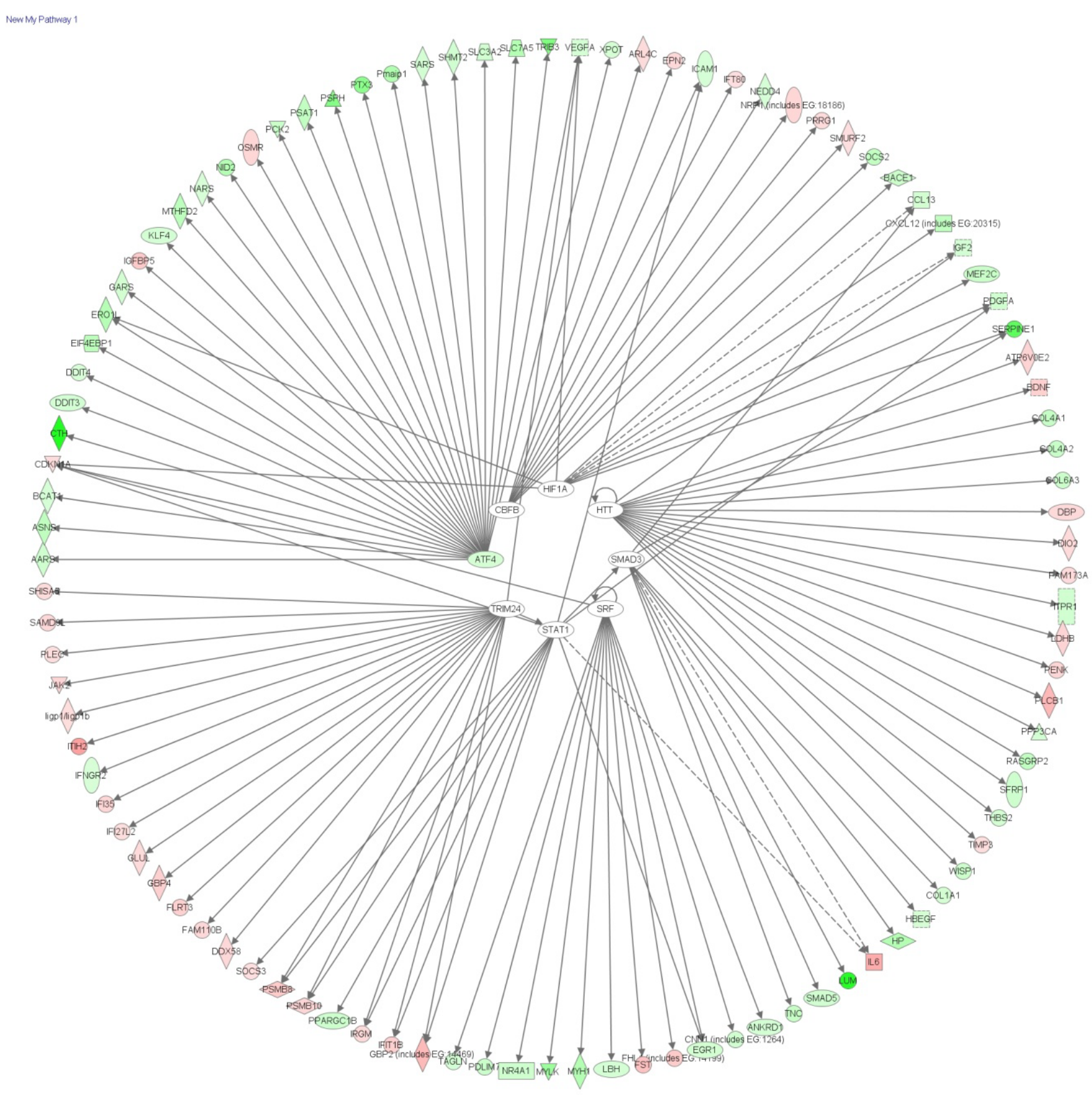

Figure 5: Ingenuity pathway analysis of transcription factors that affect differentially expressed genes in response to upregulation (TGA) of VEGF-A 
Citation: Husso T, Turunen MP, Yla-Herttuala S (2015) Regulation of VEGF-A Expression in Endothelial Cells by Transcriptional Gene Activation or Transcriptional Gene Silencing: Analysis of Genome Wide Transcriptional Response. Gene Technology 4: 122. doi: 10.4172/23296682.1000122

Page 6 of 8

proliferation, development, cell death, cell movement and antigen presentation.

STAT1 and CBFB transcription factors are activated in response to VEGF-A downregulation and SRF, SMAD3, HTT, HIF1A, ATF4 and TRIM24 transcription factors are inhibited by VEGF-A downregulation (Figure 5).

\section{Up regulation of VEGF-A}

Genes that are linked to cancer (120 genes), cardiovascular disease
(47 genes), inflammatory response (101 genes), immunological disease (43 genes) and connective tissue disorders ( 31 genes) are significantly regulated in response to the upregulation of VEGF-A in endothelial cells. Top 5 molecular and cellular functions associated are: cell growth and proliferation, cell movement, cell death, and cell-to-cell signaling and interaction.

Transcription factors ATF4, TP73, NOTCH1 and TRIM24 are activated upon the epigenetic upregulation of VEGF-A and the expression of HNF1A and TOB1 is inhibited. As in the case of

New My Pathway 2

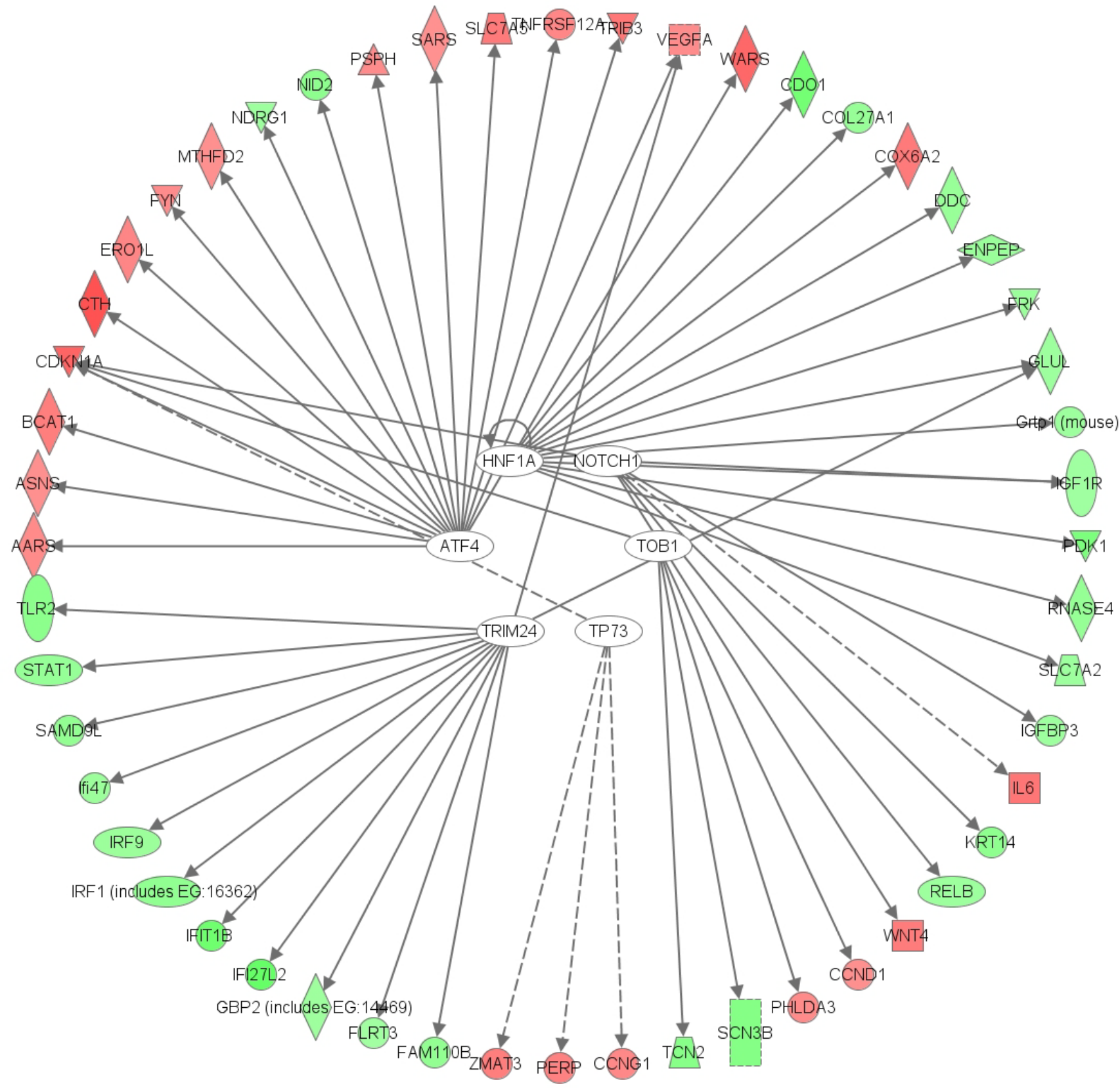

2000-2012 Ingenuity Systems, Inc. All rights reserved.

Figure 6: Ingenuity pathway analysis of transcription factors that affect differentially expressed genes in response to downregulation (TGS) of VEGF-A. 
Citation: Husso T, Turunen MP, Yla-Herttuala S (2015) Regulation of VEGF-A Expression in Endothelial Cells by Transcriptional Gene Activation or Transcriptional Gene Silencing: Analysis of Genome Wide Transcriptional Response. Gene Technology 4: 122. doi: 10.4172/23296682.1000122

Page 7 of 8

downregulation of VEGF-A, these transcription factors regulate a number of genes that are further regulating other genes and signaling pathways (Figure 6).

\section{qRT-PCR validation of differentially expressed key genes}

We validated the expression of top upregulated and downregulated genes (Tnnt2, Kdr, Ccl2, Selp, Mmp13 and Angptl4) from both groups by using qRT-PCR analysis. The expression changes were very similar for all genes as in microarray analysis. Also the differential expression of VEGF-A was confirmed using qRT-PCR analysis (Figure 7).

\section{Possible off-target effects of shRNAs}

Non-specific binding of shRNA sequences to mouse gene promoters was evaluated genome-wide by aligning the shRNA binding sequences to the mouse genome (NCBI37/mm9). We then compared the gene expression levels of the genes closest to these putative binding sites to find out if they might be regulated by the shRNAs. Importantly, the predicted off-target genes showed a very little change in their expression (Table 3).
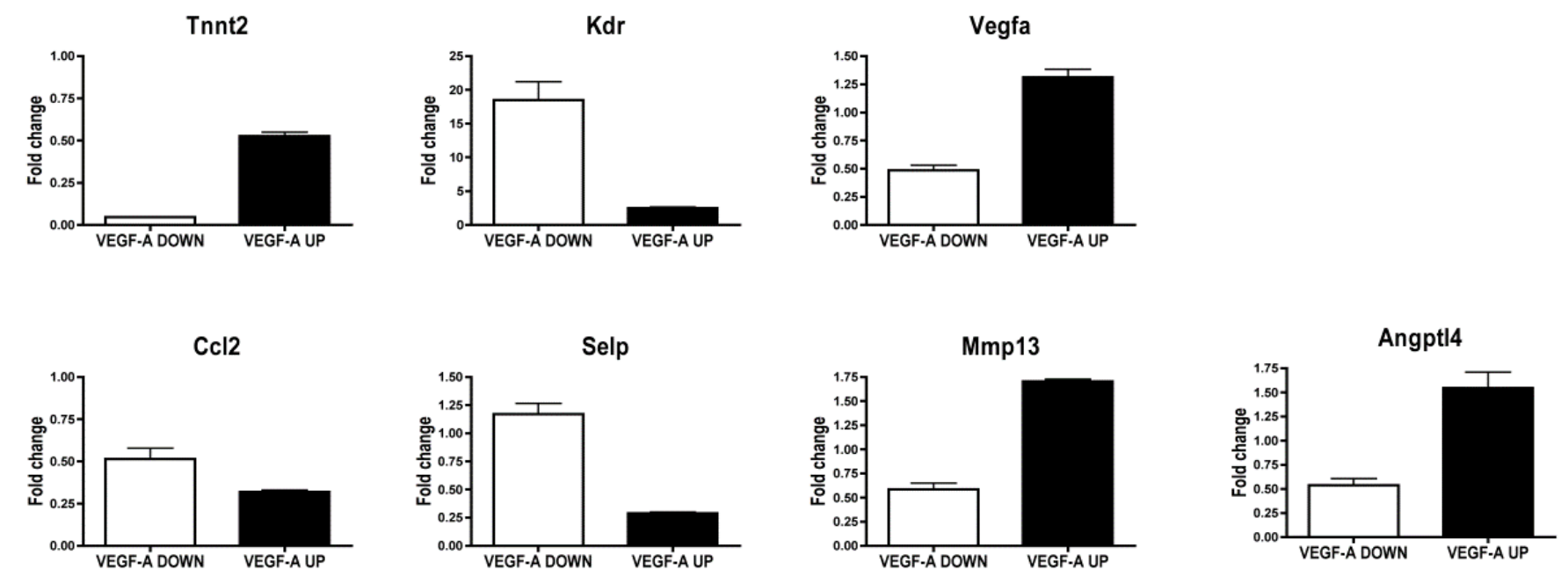

Figure 7: qRT-PCR validation of relevant target genes. Results are calculated in reference to housekeeping gene ACTB and LV control and are shown as mean \pm SD

\begin{tabular}{|c|c|c|c|c|c|}
\hline Gene & Distanceto TSS (bp) & Mismatches & shRNA & FC up & FC down \\
\hline Prkcz & 21 & $5: A>C, 12: G>T, 13: C>T$ & down & $\mathrm{NaNa}$ & $\mathrm{NaN}$ \\
\hline Vegfa & 437 & $9: C>A$ & down & 1,280103 & $-1,537277$ \\
\hline Mir3110 & 458 & $2: G>C, 4: G>C, 17: A>G$ & down & $\mathrm{NaNb}$ & $\mathrm{NaN}$ \\
\hline 3632454L22Rik & 528 & $0: T>G, 3: G>A, 10: T>C$ & up & 1,000995 & $-1,009152$ \\
\hline Vegfa & 843 & None & up & 1,280103 & $-1,537277$ \\
\hline Ces3a & 975 & $1: G>C, 12: T>G, 14: C>G$ & up & $\mathrm{NaNb}$ & $\mathrm{NaN}$ \\
\hline Rap2c & 1059 & $5: C>T, 10: C>G, 16: T>A$ & up & 1,171336 & 1,098138 \\
\hline Cldn24 & 1696 & $2: A>C, 4: A>C, 5: C>G$ & down & $\mathrm{NaNb}$ & $\mathrm{NaN}$ \\
\hline Sart3 & 2160 & $0: A>C, 9: C>T, 10: A>G$ & up & $-1,067785$ & $-1,088687$ \\
\hline Kank4 & 2165 & $0: G>C, 16: G>A$ & up & $-1,44748$ & $-1,171926$ \\
\hline Utrn & 2588 & $11: A>G, 18: A>T$ & down & 1,089009 & 1,082528 \\
\hline Defb2 & 13474 & $0: A>C, 10: C>G$ & up & $-1,003149$ & $-1,005893$ \\
\hline Neat1 & 17106 & $0: T>G, 18: T>A$ & up & $-1,045212$ & 1,462386 \\
\hline F730016J06Rik & 19668 & $1: A>C, 17: A>T$ & up & $\mathrm{NaNb}$ & $\mathrm{NaN}$ \\
\hline Dppa4 & 20860 & $0: T>C, 12: T>C$ & up & 1,048477 & 1,11234 \\
\hline Nat15 & 23343 & $7: G>T, 8: G>C$ & up & 1,026753 & 1,256342 \\
\hline Ogfod1 & 25050 & $1: T>C, 18: T>A$ & up & 1,021149 & 1,066103 \\
\hline Dpcd & 37770 & $2: C>G, 9: T>A$ & down & $-1,042219$ & 1,276962 \\
\hline
\end{tabular}

Table 3: Analysis of putative shRNA binding sites by promoter scanning. Possible off-target genes show little change in gene expression. 
Citation: Husso T, Turunen MP, Yla-Herttuala S (2015) Regulation of VEGF-A Expression in Endothelial Cells by Transcriptional Gene Activation or Transcriptional Gene Silencing: Analysis of Genome Wide Transcriptional Response. Gene Technology 4: 122. doi: 10.4172/23296682.1000122

proliferation of endothelial cells [18]. VEGF-A also activates other signaling pathways, such as Akt/PI3K [18]. VEGF-A has been associated with various diseases including cancer, cardiovascular diseases, and retinopathy [19-21]. It has been suggested as a therapeutic agent in the treatment of myocardial ischemia by overexpressing it in the heart via viral vectors [1]. Also, VEGF-A inhibition has been studied as a potential target in cancer [22].

In this study, it was found that both the activation and inhibition of VEGF-A in endothelial cells resulted in distinctive transcriptional profiles and widespread effect in the gene expression patterns of hundreds of genes. Many of the regulated genes are in turn regulated by transcription factors. One of these transcription factors, Activating transcription factor 4 (ATF4, tax-responsive enhancer element B67, CREB2) was found to be involved in the transcriptional response of both epigenetic activation and inhibition of VEGF-A. The role of CREB2 in the regulation of VEGF-A is very interesting and warrants further studies. We have previously found $\mathrm{H} 3 \mathrm{~K} 27$ to be the most important histone residue for the epigenetic regulation of mouse VEGF-A expression [8,9]. Also, we have shown that CBP/CREB interaction inhibitor abolishes the shRNA-mediated upregulation of VEGF-A. The targeting strand of this shRNA contains one mismatch ( $G$ to $T$ ) in the middle part of the sequence and this area in the murine VEGF-A promoter contains a conserved cAMP response element (CRE). CREB, which binds to CRE [23], regulates the H3K27 acetylation by binding CREB binding protein (CBP)/p300 and CREB Regulated Transcription Coactivator (CRTC) [24]. When cells were treated with CBP-CREB interaction inhibitor, the shRNA-mediated VEGF-A upregulation was reduced to the level of shRNA control [9].

It has become obvious that in addition to targeting mRNAs in the cytoplasm as in the canonical RNAi, small non-coding RNAs can target also the genome in the nucleus, and therefore all promoter areas should be checked for possible off-target effects. In this study we observed only minor gene expression changes in genes that were close to possible offtarget sites in genome. Therefore, the gene expression changes observed with both TGS and TGA were most likely due to the regulation of VEGF-A and the action of transcription factors that are regulated. Taken together, this study analyzed the transcriptional profile of mouse endothelial cells in response to epigenetic up- or downregulation of VEGF-A and provides new leads for further analysis of VEGF-A signaling and its biological actions.

\section{Data Accessibility}

GEO files for review can be found at: http://www.ncbi.nlm.nih.gov/ geo/query/GSE47444

\section{Acknowledgements}

We thank Genevia company, Finland, for assisting in bioinformatic analysis and A. Martikainen, J. Malinen and $\mathrm{H}$. Eskelinen for technical assistance. This study was supported by grants from Finnish Academy, Sigrid Juselius Foundation and ERC Advanced grant.

\section{References}

1. Yla-Herttuala S, Rissanen TT, Vajanto I, Hartikainen J (2007) Vascula endothelial growth factors: biology and current status of clinical applications in cardiovascular medicine. J Am Coll Cardiol. 49: 1015-1026.

2. Chu L-H, Lee E, Bader JS, Popel AS (2014) Angiogenesis Interactome and Time Course Microarray Data Reveal the Distinct Activation Patterns in Endothelial Cells. PLoS One. 9: e110871.

3. Zhang B, Day DS, Ho JW, Song L, Cao J, et al. (2013) A dynamic H3K27ac signature identifies VEGFA-stimulated endothelial enhancers and requires EP300 activity. Genome Res. 23: 917-927.

4. Shin JW, Huggenberger R, Detmar M (2008)Transcriptional profiling of VEGF-A and VEGF-C target genes in lymphatic endothelium reveals endothelial-specific molecule-1 as a novel mediator of lymphangiogenesis. Blood. 112: 2318-2326.

5. Davies S, Dai D, Pickett G, Thiel KW, Victoria P, et al. (2012) Effects of bevacizumab in mouse model of endometrial cancer: Defining the molecular basis for resistance. Oncol Rep. 25: 855-862.

6. Morris KV, Chan SWL, Jacobsen SE, Looney DJ (2004) Small interfering RNAinduced transcriptional gene silencing in human cells. Science. 305:1289-1292

7. Li LC, Okino ST, Zhao H, Pookot D, Place RF, et al. (2006) Small dsRNAs induce transcriptional activation in human cells. Proc Natl Acad Sci U S A. 103 $17337-1742$

8. Turunen MP, Lehtola T, Heinonen SE, Assefa GS, Korpisalo P, et al. (2009) Efficient regulation of VEGF expression by promoter-targeted lentiviral shRNAs based on epigenetic mechanism: a novel example of epigenetherapy. Circ Res. 105: 604-609.

9. Turunen MP, Husso $T$, Musthafa $H$, Laidinen $S$, Dragneva $G$, et al (2014) Epigenetic upregulation of endogenous VEGF-A reduces myocardial infarct size in mice. PLoS One. 9: e89979.

10. Mäkinen PI, Koponen JK, Karkkäinen AM, Malm TM, Pulkkinen KH, et al. (2006) Stable RNA interference: comparison of $\mathrm{U} 6$ and $\mathrm{H} 1$ promoters in endothelial cells and in mouse brain. J Gene Med. 8:433-441.

11. Storey JD, Tibshirani R (2003) Statistical significance for genomewide studies. Proc Natl Acad Sci U S A. 100: 9440-9445.

12. Senger DR, Ledbetter SR, Claffey KP, Papadopoulos-Sergiou a, Peruzzi CA et al. (1996)Stimulation of endothelial cell migration by vascular permeability factor/vascular endothelial growth factor through cooperative mechanisms involving the alphavbeta3 integrin, osteopontin, and thrombin. Am J Pathol. 149: 293-305

13. Gupta K, Kshirsagar S, Li W, Gui L, Ramakrishnan S, et al. (1999) VEGF prevents apoptosis of human microvascular endothelial cells via opposing effects on MAPK/ERK and SAPK/JNK signaling. Exp Cell Res. 247: 495-504.

14. Ferrara N (2004) Vascular endothelial growth factor: Basic science and clinical progress. Endocr Rev. 25: 581-611.

15. Pugh CW, Ratcliffe PJ (2003) Regulation of angiogenesis by hypoxia: role of the HIF system. Nat Med. 9: 677-84

16. Olsson AK, Dimberg A, Kreuger J, Claesson Welsh L (2006) VEGF recepto signalling - in control of vascular function. Nat Rev Mol Cell Biol. 7: 359-371.

17. Shibuya M (2006) Vascular endothelial growth factor receptor-1 (VEGFR-1/FIt1): a dual regulator for angiogenesis. Angiogenesis. 9: 225-230.

18. Takahashi T, Ueno H, Shibuya M (1999) VEGF activates protein kinase C-dependent, but Ras-independent Raf-MEK-MAP kinase pathway for DNA synthesis in primary endothelial cells. Oncogene.18: 2221-2230.

19. Carmeliet P, Jain RK (2000) Angiogenesis in cancer and other diseases. Nature. 407: 249-257

20. Yla Herttuala S, Rissanen TT, Vajanto I, Hartikainen J (2007) Vascular endothelial growth factors: biology and current status of clinical applications in cardiovascular medicine. J Am Coll Cardiol. 49: 1015-1026.

21. Benjamin LE (2001) Glucose, VEGF-A, and diabetic complications. Am J Pathol. 158: 1181-1184.

22. Carmeliet $P$ (2005) VEGF as a key mediator of angiogenesis in cancer. Oncology. 69 Suppl 3:4-10

23. Zhang X, Odom DT, Koo S-H, Conkright MD, Canettieri G, et al. (2005) Genome-wide analysis of cAMP-response element binding protein occupancy, phosphorylation, and target gene activation in human tissues. Proc Natl Acad Sci USA. 102: 4459-4464.

24. Sekeres MJ, Mercaldo V, Richards B, Sargin D, Mahadevan V, et al. (2012) Increasing CRTC1 function in the dentate gyrus during memory formation or reactivation increases memory strength without compromising memory quality. J Neurosci. 32: 17857-17868. 